\title{
Quasars from LSST as dark energy tracers: first steps*
}

\author{
Mary Loli Martínez-Aldama \\ Center for Theoretical Physics, Polish Academy of Sciences \\ E-mail: mmary@cft.edu.pl
}

\section{Swayamtrupta Panda}

Center for Theoretical Physics, Polish Academy of Sciences

Nicolaus Copernicus Astronomical Center, Polish Academy of Sciences

panda@cft.edu.pl

\section{Bożena Czerny}

Center for Theoretical Physics, Polish Academy of Sciences

bcz@cft.edu.pl

\section{Michal Zajaček}

Center for Theoretical Physics, Polish Academy of Sciences

zajacek@cft.edu.pl

In the near future, new surveys promise a significant increase in the number of quasars (QSO) at large redshifts. This will help to constrain the dark energy models using quasars. The Large Synoptic Survey Telescope (LSST) will cover over 10 million QSO in six photometric bands during its 10-year run. QSO will be monitored and subsequently analyzed using the photometric reverberation mapping $(\mathrm{RM})$ technique. In low-redshift quasars, the combination of reverberation-mapped and spectroscopic results have provided important progress. However, there are still some facts which have to be taken into account for future results. It has been found that super-Eddington sources show time delays shorter than the expected from the well-known Radius-Luminosity $\left(R_{\mathrm{H} \beta}-L_{5100}\right)$ relation. Using a sample of $117 \mathrm{H} \beta$ reverberation-mapped AGN with $0.02<z<0.9$, we propose a correction by the accretion rate effect recovering the classical $R_{\mathrm{H} \beta}-L_{5100}$ relation. We determined the cosmological constants, which are in agreement with $\Lambda$-Cold Dark Matter model within $2 \sigma$ confidence level, which is still not suitable for testing possible departures from the standard model. Upcoming LSST data will decrease the uncertainties in the dark energy determination using reverberation-mapped sources, particularly at high redshifts. We show the first steps in the modeling of the expected light curves for $\mathrm{H} \beta$ and Mg II.

Multifrequency Behaviour of High Energy Cosmic Sources - XIII - MULTIF2019

3-8 June 2019

Palermo, Italy

\footnotetext{
*on behalf of the LSST AGN SC Collaboration

† Speaker.

$¥$ Acknowledgements. The project was partially supported by National Science Centre, Poland, grant No. 2017/26/A/ST9/00756 (Maestro 9) and MNiSW grant DIR/WK/2018/12.
} 


\section{Introduction}

Understanding the behavior of dark energy is one of the most challenging problems in physics and astrophysics nowadays. Dark energy is responsible for the accelerated Universe expansion, indicated by precise measurements based on Supernovae Ia, Cosmic Microwave Background (CMB), Baryon Acoustic Oscillations (BAO) and weak lensing. The most recent values of the cosmological constants: $\Omega_{m}=0.3111 \pm 0.0056, \Omega_{\Lambda}=0.6889 \pm 0.0056$ and $H o=67.66 \pm 0.42 \mathrm{~km} \mathrm{~s}^{-1} \mathrm{Mpc}^{-1}$ (Planck Collaboration, 2018), suggest a flat geometry which satisfies the $\Lambda$-cold dark matter model $(\Lambda \mathrm{CDM})$. Cosmological estimations are done using two observable indicators: standard rules and standard candles. Standard rulers are sources with a known angular size, which is converted to a physical size and it is then possible to determine the distance to the source. It is the technique used on the BAO estimations. On the other hand, standard candles are objects where the intrinsic luminosity is known and the luminosity distance $\left(D_{\mathrm{L}}\right)$ can be inferred. A classical example is Supernovae Ia (SN Ia), which have constrained cosmological models up to $z \sim 1.4$. Higher redshift SN Ia are rare, and their use can be evolutionary biased.

The necessity of sources with larger redshift ranges for testing the cosmological models will be provided by the next generation of surveys. The Large Synoptic Survey Telescope $(L S S T)^{1}$ will observe over 10 million quasars in six photometric bands during its 10-year run and analyzed using the photometric reverberation mapping technique. Reverberation mapping is based on the time delay between the emission line and the continuum variations. This technique has been applied to around $100 \mathrm{AGN}$ and quasars mainly in the $\mathrm{H} \beta$ region, although there are some measurements using Civ $\lambda 1549$ and Mg II (e.g Grier et al., 2019; Czerny et al., 2019). The low number of reverberation-mapped sources is mainly due to the required long-term monitoring time, which will be solved by LSST.

Reverberation mapping has provided important results on AGN and quasars physics, which are summarized in Section 2. This technique has potential use in cosmology since it allows to determine the distance to the source in an independent way, applying the relation between the size of the broad line region $\left(R_{\mathrm{H} \beta}\right)$ and the monochromatic luminosity $\left(L_{\lambda}\right)$. However, it has recently been found that high Eddington sources show a departure from $R_{\mathrm{H} \beta}-L_{5100}$ relation in the optical range (Section 3) and are associated with the smallest continuum variations (Section 4). We proposed a correction by the accretion rate effect (Section 3), which permit us to estimate the cosmological constants within $2 \sigma$ confidence level (Section 5). The large uncertainties would be due to the low number of sources and the different techniques employed in the literature to estimate the time delay between the emission line and the continuum. This correction proposed by us needs to be taken into account for the future $L S S T$ results. In Section 6 we present the first steps in modeling of the light curves expected for $\mathrm{H} \beta$ and $\mathrm{Mg}$ II.

\section{Radius-Luminosity relation}

It has been observed that broad emission lines respond to the continuum variations with a time delay $\left(\tau_{\text {obs }}\right)$ of days or weeks. This time is directly related to the light travel across the broad-line region (BLR), i.e., $\tau_{\mathrm{obs}}=R_{\mathrm{H} \beta} / c$, where $R_{\mathrm{H} \beta}$ is the size of the BLR and $c$ is the speed

\footnotetext{
${ }^{1}$ Also known as the Vera Rubin Survey Telescope, for more details see https://www.lsst.org/
} 
of light. Reverberation mapping (RM) is based on the monitoring of the source to get $\tau_{\text {obs. }}$. It has provided information about the stratification of the BLR. For example, the time delay shown by high-ionization emission lines (HILs), like CIV $\lambda 1549$, is smaller than that shown by lowionization lines (LILs), like $\mathrm{H} \beta$. It means that HILs are emitted in a closer zone to the central continuum source.

The most important result of RM is the Radius-Luminosity relation. In the optical range (Bentz et al., 2013), it is given by:

$$
\log \left(\frac{R_{\mathrm{H} \beta}}{11 \mathrm{t}-\text { day }}\right)=(1.527 \pm 0.31)+0.533_{-0.033}^{+0.035} \log \left(\frac{L_{5100}}{10^{44} L_{\odot}}\right) .
$$

By knowing the luminosity of the source, we can determine the size of the BLR and thus other properties like the black hole mass and the accretion rate.

Using a sample of $117 \mathrm{H} \beta$ reverberation-mapped sources, we built a $R_{\mathrm{H} \beta}-L_{5100}$ diagram, which is shown in the left panel of Figure 1. To estimate the black hole mass $\left(M_{\mathrm{BH}}\right)$, we considered the virial factor anti-correlated with the full-width at half maximum (FWHM) of the emission line $\left(f_{\mathrm{BLR}}\right)$ proposed by Mejía-Restrepo et al. (2018), which includes a correction for the orientation effect. Accretion rate is estimated considering the dimensionless accretion rate $\left(\dot{\mathscr{M}}^{\mathrm{c}}\right)$ described by Du et al. (2015). A similar result was also found by Yu et al. (2019). The assumptions in the estimation of these parameters could include some bias and caveats in our analysis, which are discussed in the Sections 3.1 $M_{\mathrm{BH}}$ and $\dot{\mathscr{M}}^{\mathrm{c}}$ values, and details of the sample are reported in Martínez-Aldama et al. (2019).

The left panel of Figure 1 illustrates the variation of the dimensionless accretion rate through $R_{\mathrm{H} \beta}-L_{5100}$ diagram. Sources with the largest $\dot{\mathscr{M}}^{\mathrm{c}}$ values show the largest departures from the $R_{\mathrm{H} \beta}-L_{5100}$ relation, i.e., their time delays are smaller than that predicted by the $R_{\mathrm{H} \beta}-L_{5100}$ relation (Du et al., 2015, 2018). Super-Eddington sources show a particular behavior compared with the rest of the AGN. Wang et al. (2014b) found that their continuum is produced by a slim disk. Their BLR tends to show large densities $\left(n_{H} \sim 10^{13} \mathrm{~cm}^{-3}\right)$ and low-ionization parameters ( $\log U<-2$ ) (Negrete et al., 2012). Also, some spectroscopic features are characteristic of this kind of sources, like the strong intensity of very low-ionization lines (e.g. optical FeII) or the blue asymmetries in the HIL profiles (Martínez-Aldama et al., 2018). All these features are directly related to the high accretion rate shown by these sources, which is also responsible of the departure for the $R_{\mathrm{H} \beta}-L_{5100}$ relation.

\section{Departure from the $R_{\mathrm{H} \beta}-L_{5100}$ relation}

The departure from the $R_{\mathrm{H} \beta}-L_{5100}$ relation is estimated by the parameter $\Delta R_{\mathrm{H} \beta}$ defined as $\Delta R_{\mathrm{H} \beta}=\log \left(\frac{\tau_{\mathrm{obs}}}{\tau_{\mathrm{H} \beta_{\mathrm{R}-\mathrm{L}}}}\right)$, where $\tau_{\mathrm{H} \beta_{\mathrm{R}-\mathrm{L}}}$ is the time delay expected from the $R_{\mathrm{H} \beta}-L_{5100}$ relation and can be estimated from the Equation 2.1. However, since the $M_{\mathrm{BH}}$ and $\dot{\mathscr{M}}^{\mathrm{c}}$ are estimated from $\tau_{\text {obs }}$, a self-correlation is introduced in the parameter $\Delta R_{\mathrm{H} \beta}$. We expect that the departure observed in the $R_{\mathrm{H} \beta}-L_{5100}$ relation is physically related to the Eddington ratio, but self-correlation likely affects quantitative estimates of the trend, and thus the results presented in this work. A deep discussion is included in Section 3.2. 

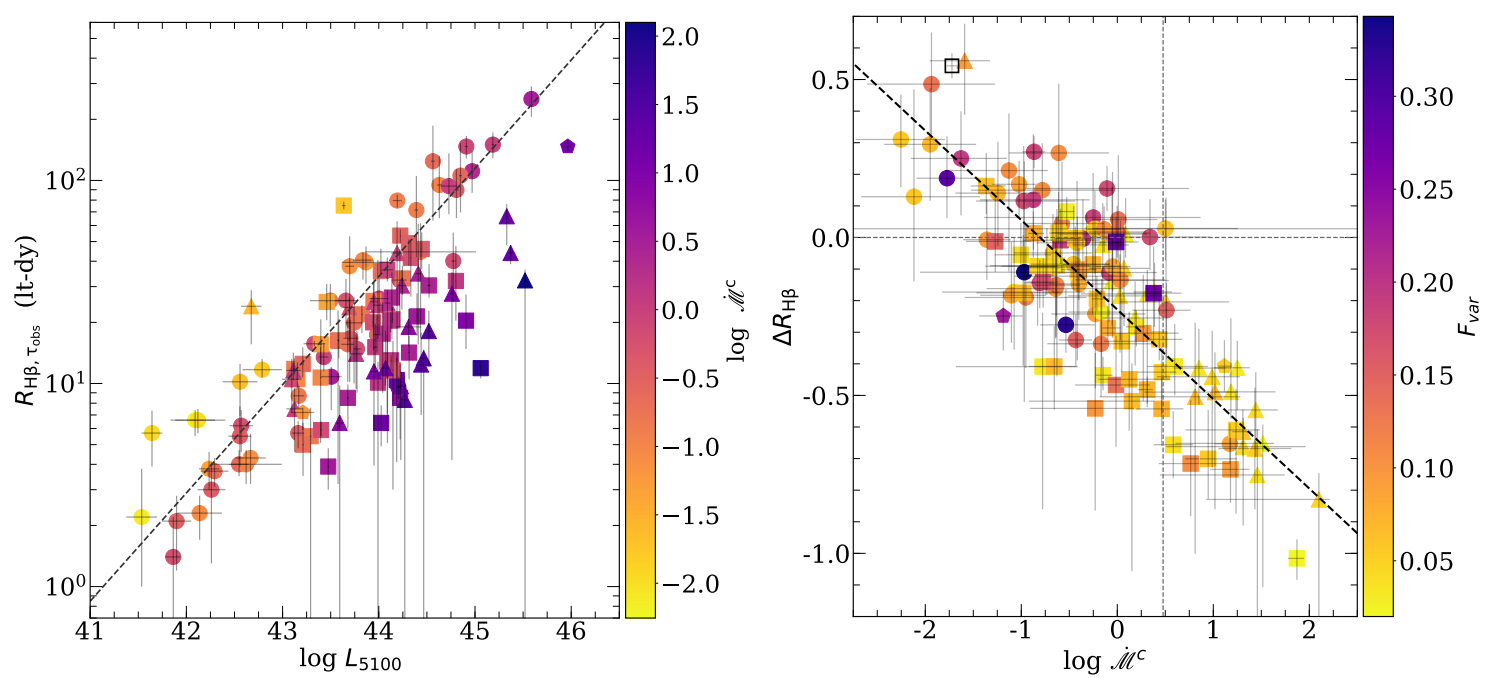

Figure 1: LEFT PANEL: $R_{H \beta}-L_{5100}$ relation for SEAMBH (triangles), SDSS-RM (squares), Bentz Collection (circles), NGC5548 and 3C 273 (pentagons). Colors indicate the variation in dimensionless accretion rate, $\dot{\mathscr{M}}^{\mathrm{c}}$. Dashed black line corresponds to the expected $R_{H \beta}-L_{5100}$ relation from Bentz et al. (2013) (Equation 2.1). RIGHT PANEL: Relation between $\Delta R_{\mathrm{H} \beta}$ and $\dot{\mathscr{M}}^{\mathrm{c}}$. Marker colors indicate the variation in $F_{\text {var }}$ at $5100 \AA$. In both panels, the parameters have been estimated with an anti-correlated virial factor, $f_{\mathrm{BLR}}^{\mathrm{c}}$. SEAMBH stands for super-Eddington accreting massive black holes (Wang et al., 2014a), the SDSS-RM sample is taken from Grier et al. (2017) and the Bentz Collection is described in Section 2 in Martínez-Aldama et al. (2019).

In right panel of Figure 1 is shown the behavior of $\Delta R_{\mathrm{H} \beta}$ as a function of the $\dot{\mathscr{M}}^{\text {c }}$. There is a strong relation between these two parameters, which is supported by the Pearson coefficient $(P=0.822)$ and $r m s(0.172)$ values. In order to describe this relation, we performed an orthogonal linear fit, which is given by:

$$
\Delta R_{\mathrm{H} \beta, \mathscr{M}^{\mathrm{c}}}=(-0.283 \pm 0.017) \log \dot{\mathscr{M}}^{\mathrm{c}}+(-0.228 \pm 0.016)
$$

Therefore, the largest accretion rates correspond to the largest departures. With this relation we can correct the observed time delay using the relation:

$$
\tau_{\text {corr }}\left(\dot{\mathscr{M}}^{c}\right)=10^{-\Delta R_{\mathrm{H} \beta}\left(\dot{\mathscr{M}}^{\mathrm{c}}\right)} \cdot \tau_{\mathrm{obs}} .
$$

With this correction, we recover the low scatter $\left(\sigma_{\text {corr }}=0.396\right)$ along the $R_{\mathrm{H} \beta}-L_{5100}$ relation as is shown in the Figure 2.

\subsection{Remarks on $M_{\mathrm{BH}}$ and $\dot{\mathscr{M}}^{\mathrm{c}}$ estimations}

Black hole mass is estimated from the relation: $M_{\mathrm{BH}}=f_{\mathrm{BLR}} \frac{R_{\mathrm{H} \beta} F W H M^{2}}{G}$, where $f_{\mathrm{BLR}}$ is the virial factor which includes information about the dynamics, structure and orientation of the BLR. Many formalisms have been proposed to get a general scheme of its behavior (e.g. Onken et al., 2004; Collin et al., 2006; Woo et al., 2015; Yu et al., 2019, and references therein), however, the 


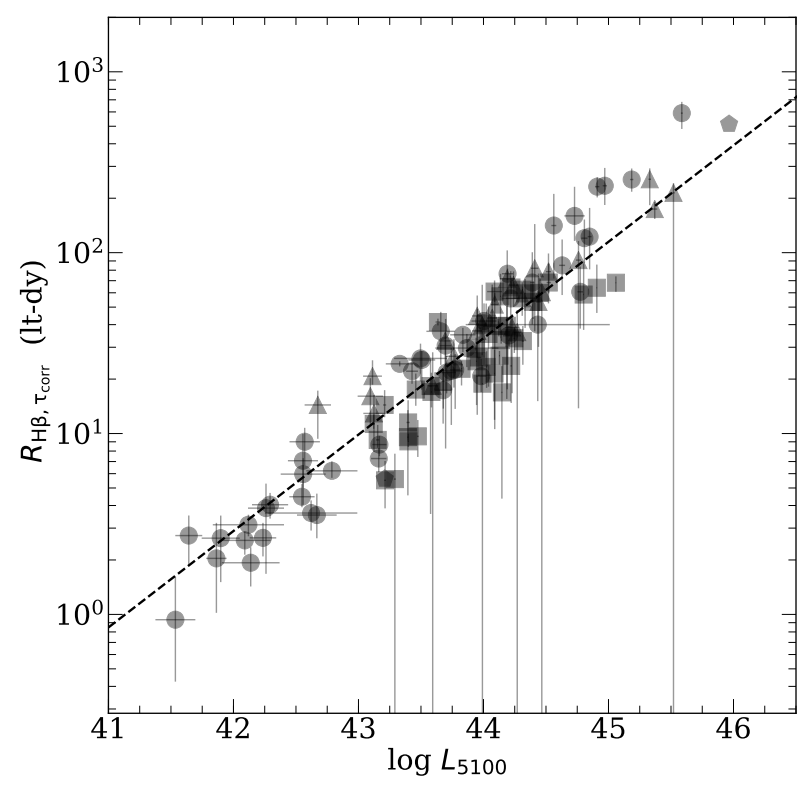

Figure 2: $R_{\mathrm{H} \beta}-L_{5100}$ relation corrected by the dimensionless accretion rate effect. Symbols are the same as Figure 1.

large diversity of AGN properties complicate the scenario. The virial factor uncertainty affects directly the black hole mass determination introducing an error by a factor 2-3.

The proper way to calibrate the virial factor is by the comparison with an independent method to get the black hole mass. Typically, the relation between the $M_{\mathrm{BH}}$ and the bulge or spheroid stellar velocity dispersion $\left(\sigma_{*}\right)$, the relation $M_{\mathrm{BH}}-\sigma_{*}$ (e.g. Ferrarese and Merritt, 2000; Gültekin et al., 2009; Woo et al., 2015) is used. Although the estimation is based on the rms spectrum of the reverberation-mapped AGN, which is not strongly affected by orientation effects, there are still large uncertainties when it is applied to the general AGN population. Also, there is a lack of super-Eddington sources in the estimations.

Collin et al. (2006) found that the virial factor changes according to the shape of the profile (Gaussian or Lorentzian). Sources with narrower profiles would be associated with virial factors larger than broad profiles. The viral factor proposed by Mejía-Restrepo et al. (2018) shows this behavior, $f_{\mathrm{BLR}}^{\mathrm{c}}=\left(\frac{\mathrm{FWHM}_{\mathrm{obs}}}{4550 \pm 1000}\right)^{-1.17}$ (expression for the $\mathrm{H} \beta$ emission line). Recently, an independent analysis done by Yu et al. (2019) provided a similar expression for the virial factor, which supports the selection for our analysis.

However, $f_{\mathrm{BLR}}^{\mathrm{c}}$ has some caveats. It has been performed using a sample with a predominance of broad profiles (FWHM $>3000 \mathrm{~km} \mathrm{~s}^{-1}$ ), then the results could change with the inclusion of a large number of sources with narrower profiles. One-third of our sample has narrow profiles (FWHM $<2000 \mathrm{~km} \mathrm{~s}^{-1}$ ), which also corresponds to the super-Eddington sources. This fact would change $\dot{\mathscr{M}}^{\text {c }}$ values and the correction proposed by the accretion rate effect (Equation 3.1).

The selection of a variable virial factor recovers in some sense the orientation effect, according to Storchi-Bergmann et al. (2017) (see also Panda et al. 2019b). We repeated the analysis using a fixed virial factor, $f_{\mathrm{BLR}}=1$, which is typically used in the single-back hole mass estimations. 
The scatter shown in the relation between $\Delta R_{\mathrm{H} \beta}$ and the dimensionless accretion rate is larger than that shown using a virial factor anti-correlated with the line FWHM, $r m s=0.172$ vs. $r m s=$ 0.243 respectively. Also, the relation is weaker according to Pearson coefficient value $(P=0.572)$. Therefore, it suggests that a variable virial factor corrects the orientation effect.

\subsection{Remarks on the $\Delta R_{\mathrm{H} \beta}$ parameter}

Our analysis is based on the correlation between the parameter $\Delta R_{\mathrm{H} \beta}$ and $\dot{\mathscr{M}}^{\mathrm{c}}$, which is used to correct the departures shown in the classic $R_{\mathrm{H} \beta}-L_{5100}$ relation. With this correction, we can recover the low scatter in the $R_{\mathrm{H} \beta}-L_{5100}$ relation and use it in cosmological analysis (Section 5). However, since $\dot{\mathscr{M}}^{\mathrm{c}}$ is estimate from the $M_{\mathrm{BH}}$ and, in turn from $\tau_{\text {obs }}$, a self-correlation is presented in the relation $\Delta R_{\mathrm{H} \beta}-\dot{\mathscr{M}}^{\mathrm{c}}$. Since it cannot be avoid, it could call into question the veracity of the accretion rate effect in the $R_{\mathrm{H} \beta}-L_{5100}$ and the results presented in this work.

For clarifying the accretion rate effect in the departures from the $R_{\mathrm{H} \beta}-L_{5100}$ relation, it requires an independent parameter which correlates with $\dot{\mathscr{M}}^{\mathrm{c}}$. Several analysis have shown that the parameter $\mathrm{R}_{\mathrm{FeII}}$, which measures the intensity of the optical FeII weighed by the $\mathrm{H} \beta$ intensity, is driven by the accretion rate or Eddington ratio (Marziani et al., 2001; Shen and Ho, 2014). Thus, the super-Eddington sources tend to be related with strong FeII emitters, and vice-versa. Based on this fact, Du and Wang (2019) and Yu et al. (2020a) explored the possibility to decrease the scatter in the $R_{\mathrm{H} \beta}-L_{5100}$ relation using $\mathrm{R}_{\mathrm{FeII}}$ and thus skip the self-correlation presented by $\dot{\mathscr{M}}^{\mathrm{c}}$. They proposed a new radius-luminosity relation with the next form: $\log \left(R_{H \beta} /\right.$ lt-day $)=\alpha+\beta \log L_{5100}+\gamma \mathrm{R}_{\mathrm{FeII}}$. The inclusion of $\mathrm{R}_{\mathrm{FeII}}$ decreases significantly the scatter in the classical $R_{\mathrm{H} \beta}-L_{5100}$ relation with similar scatter to the one find by us using $\dot{\mathscr{M}}^{\mathrm{c}}$. It shows that $R_{\mathrm{H} \beta}-L_{5100}$ relation is affected by the accretion rate, which in some sense support the results presented in this work.

\section{Variability}

The colors in the right panel of Figure 1 show the behavior of $F_{\text {var }}$, which estimates the rms of the intrinsic variability relative to the mean flux (Rodríguez-Pascual et al., 1997). $F_{\text {var }}$ mimics the behavior of the amplitude of variability. In the Figure an anti-correlation between $F_{\text {var }}$ and the dimensionless accretion rate is observed. The Spearman coefficient $\left(\rho_{s}=-0.374\right.$ with a probability of $p=1.6 \times 10^{-5}$ ) indicates a weak relation. A similar correlation has been found by several authors in larger samples (e.g. Sánchez-Sáez et al., 2018, and references therein). LSST will be able to measure the variability properties like $F_{\text {var }}$, which can be used as a tool in the identification of the physical properties of the AGNs.

\section{Hubble diagram}

Using the corrected time delay by the accretion rate effect $\left(\tau_{\text {corr }}\right)$ and the luminosity at $5100 \AA$ $\left(L_{5100}\right)$, we estimate the luminosity distance using the equation $D_{\mathrm{L}}=\left(\frac{L_{5100}}{4 \pi F_{5100}}\right)^{1 / 2}$. Then, we built a Hubble diagram, which is shown in the left panel of Figure 3. In the diagram, we also show the luminosity distance expected from the classical $\Lambda$ CDM model with parameters: $H_{0}=67 \mathrm{~km} \mathrm{~s}^{-1}$ $\mathrm{Mpc}^{-1}, \Omega_{\Lambda}=0.68, \Omega_{m}=0.32$ (black line). We also mark the luminosity distance for redshift bins 
of $\Delta z=0.1$ (red symbols), these values are only included for visualization, since the number of points are not enough for statistical analysis.
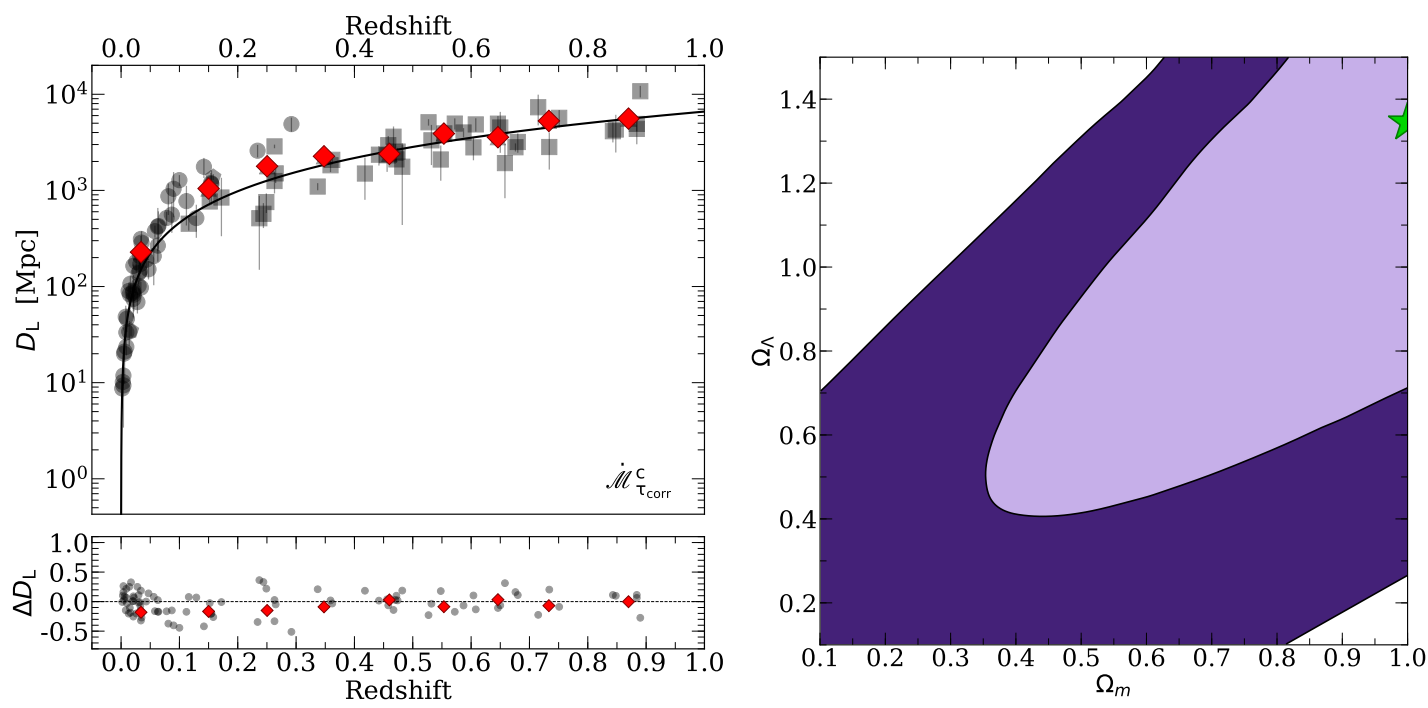

Figure 3: LEFT PANEL: Hubble diagram obtained from the time delay corrected by the dimensionless accretion rate effect. Gray symbols are the same as Fig. 1. Red symbols correspond to the mean of the luminosity distance in redshift bins of $\Delta z=0.1$. The black line indicates the expected luminosity distance based on the standard $\Lambda \mathrm{CDM}$ model. The bottom panel shows the difference between the expected luminosity distance and the observed one. RIGHT PANEL: $\chi^{2}$ behavior in the $\Omega_{m}-\Omega_{\Lambda}$ space for the full sample. Contours correspond to $1 \sigma$ and $2 \sigma$ confidence levels, respectively. Green symbol marks the minimum $\chi^{2}$ value.

In order to get a determination of the cosmological parameters, we estimate the best cosmological model. We assumed a standard $\Lambda$ CDM model, and the value of the Hubble constant, $H_{0}=67$ $\mathrm{km} \mathrm{s}^{-1} \mathrm{Mpc}^{-1}$, and search for the minimum value (best fitting) using the function:

$$
\chi^{2}=\sum_{i=1}^{N} \frac{\left(\log \left(D_{\mathrm{L}, \bmod }^{i}\right)-\log \left(D_{\mathrm{L}, \mathrm{obs}}^{i}\right)\right)^{2}}{\left(\log \left(1+b_{i}\right)^{2}+\sigma^{2}\right)},
$$

where $N$ is the total number of sources in the sample, $b_{i}$ is the relative error in the luminosity distance determination and $\sigma$ is the dispersion in the sample described by Risaliti and Lusso (2015). The best fit is shown in the right panel of Figure 3 (green symbol), which is consistent within $2 \sigma$ confidence level with the standard $\Lambda \mathrm{CDM}$ model. However, the accuracy is not enough for cosmological results.

Since the main source of error is the time delay uncertainty, the main challenge is reduced it. In order to get a small lag uncertainty are required a high time resolution, long-monitoring time, homogeneous and high $\mathrm{S} / \mathrm{N}$ data and, high spectral resolution. However, the uncertainty is also depend on the method used to determine the time delay. Typically, JAVELIN ( $\mathrm{Zu}$ et al., 2011a) provides smaller uncertainties compared with the ones given by other methods like the Interpolated cross-correlation function (ICCF) or the Continuum REprocessing AGN MCMC (CREAM). Recently. Yu et al. (2020b) based on simulated lightcurves for five long-monitoring AGN provided a 
comparison between the uncertainties given by JAVELIN and ICCF exploring several systematic errors. They found that ICCF overestimates the lag uncertainties while JAVELIN provides a better estimation under the same systematic errors. Therefore, the combination of long-monitoring time and high-quality observations plus the implementation of JAVELIN should provide small uncertainties. The majority of the time delay reported in this work are based on ICCF method. then, if JAVELIN provides smaller uncertainties, it could be an option to decrease the uncertainties in cosmological analysis .

\section{Prospect for $L S S T$}

LSST (Ivezić et al., 2019) is a $8.4 \mathrm{~m}$ telescope with a state-of-the-art 3.2 Gigapixel flat-focal array camera that will allow to perform rapid scan of the sky with 15 seconds exposure and thus providing a moving array of color images of objects that change. Every night, $L S S T$ will monitor $\sim 75$ million AGNs and is estimated to detect $\sim 300+$ million AGNs in the $\sim 18000 \mathrm{deg}^{2}$ mainsurvey area (Luo et al., 2017).

LSST is a photometric project but the 6-channel photometry can be effectively used for the purpose of reverberation mapping and estimation of time delays. We present some preliminary results from our code in development which allows to produce mock light curves and recover the time delays. The code takes into consideration several key parameters to produce these light curves, namely - (1) the campaign duration of the instrument (10 years); (2) number of visits per photometric band; (3) the photometric accuracy $(0.01-0.1 \mathrm{mag})^{2}$; (4) black hole mass distribution ${ }^{3}$; (5) luminosity distribution ${ }^{4}$; (6) redshift distribution ${ }^{5}$; and (7) line equivalent widths (EWs) consistent with SDSS quasar catalogue (Shen et al., 2011). We create continuum stochastic lightcurve for a quasar of an assumed magnitude and redshift from AGN power spectrum with Timmer-Koenig algorithm (Timmer and Koenig, 1995). The code takes as an input a first estimate for the time delay measurement. We utilize the standard $R_{\mathrm{H} \beta}-L_{5100}$ relation (Bentz et al., 2013) to estimate this value. In the current version of the code, the results for the photometric reverberation method are estimated by adopting only 2 photometric channels at a time and the time delay is estimated using the $\chi^{2}$ method. We account for the contamination in the emission line $(\mathrm{H} \beta, \mathrm{MgII})$ as well as the in the continuum. The code also incorporates the FeII pseudo-continuum and contamination from starlight i.e. stellar contribution.

Since $\mathrm{H} \beta$ and $\mathrm{Mg}$ II are the typical virial estimators at low and high redshift respectively, we performed a first test of their simulated lightcurves. In the upper panels of each of Figures 4 and 5, we show the variation in the simulated lightcurves for $\mathrm{H} \beta$ as a function of the redshift and luminosity. Here, the time axis refers to the source monitoring time (in days). In the lower panels of each of Figures 4 and 5, we show the corresponding time delay distributions. These time delays are obtained using the $\chi^{2}$ method, where, we have first assumed that the delay is null. We interpolate between the data points for the two channels and starting with an assumed shift between the two interpolated light curves to be null, we loop over the light curves to estimate the best shift over the

\footnotetext{
${ }^{2}$ these values are adopted from Ivezić et al. (2019)

${ }^{3}$ the results shown here are for a representative black hole mass, $M_{B H}=10^{8} M_{\odot}$

${ }^{4}$ the results shown here are for two representative cases of bolometric luminosity, $\mathrm{L}_{\mathrm{bol}}=10^{45}$ and $10^{46} \mathrm{erg} \mathrm{s}^{-1}$.

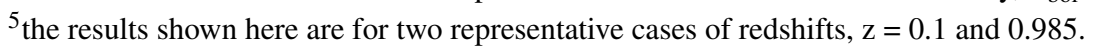


distribution using cross-correlation. This best shift at each instance is represented in the form of time-delay histograms. In Figure 5, we show the variation in the lightcurves for two emission lines $(\mathrm{MgII}$ and $\mathrm{H} \beta$ ) at the same redshift. Similar to the Figure 4, we illustrate the lightcurves for two cases of luminosities.

The density of the photometric data-points is adopted from Ivezić et al. (2019). An increase in the density of the points, both in the continuum channels and the line channels, leads to better recovery of the time delay measurements (Panda et al., 2019a), i.e., in the Deep-Drilling Fields (Brandt et al., 2018). The power spectral distribution for these lightcurves is assumed to have the low-frequency break corresponding to 2000 days.
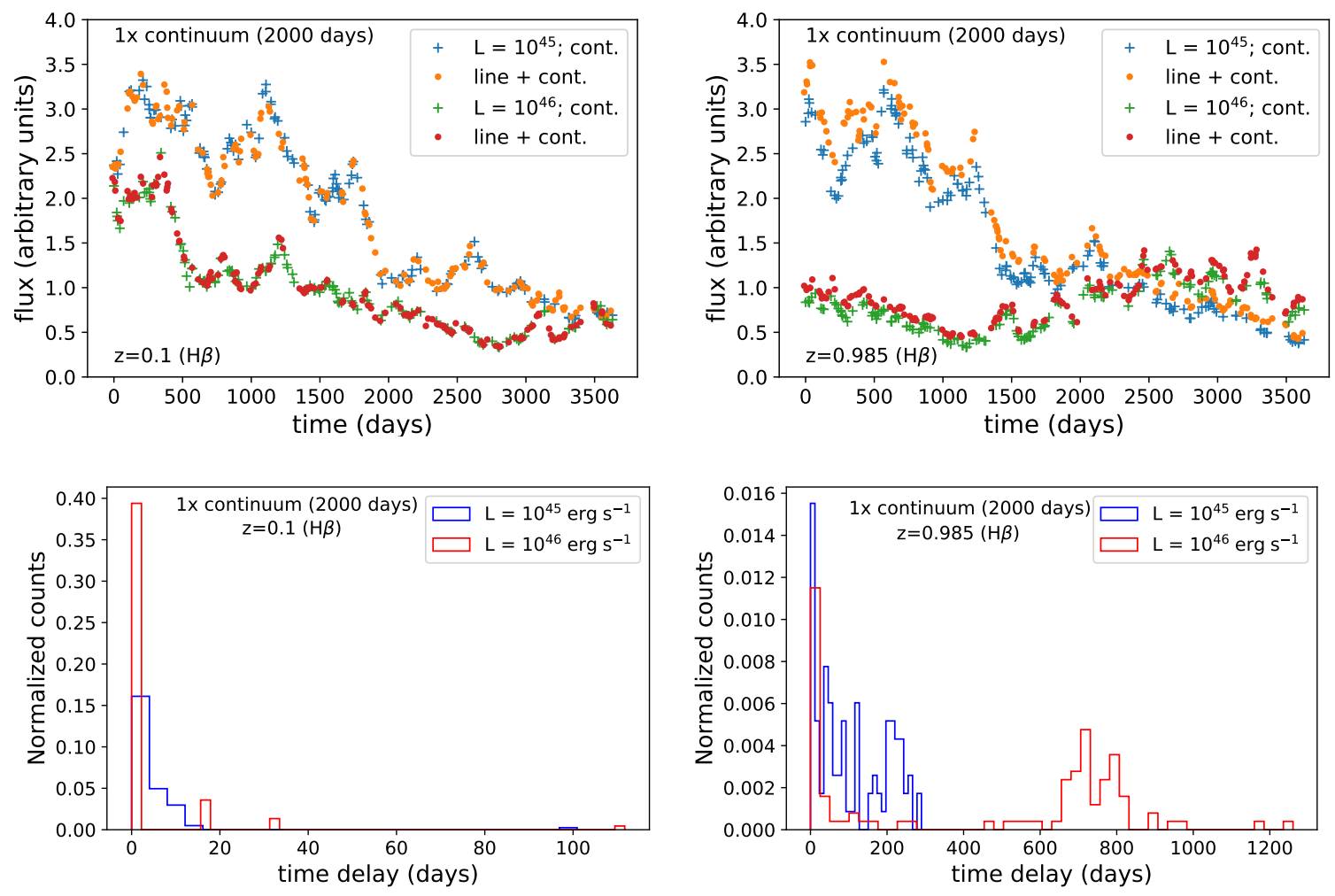

Figure 4: Preliminary lightcurves for $\mathrm{H} \beta$ at $z=0.1$ (UPPER-LEFT) and $z=0.985$ (UPPERRIGHT). The lightcurves are generated using Timmer-Koenig method (Timmer and Koenig, 1995) and convolved with photometric data distribution expected from LSST (Ivezić et al., 2019) with arbitrary normalization. Two cases of luminosity $\left(10^{45}\right.$ and $\left.10^{46} \mathrm{erg} \mathrm{s}^{-1}\right)$ are shown, each for the continuum and the line. The time axis refers to the monitoring time (in days) in the upper panels. The corresponding time delay distributions are shown in the lower panels.

\section{Conclusions and work in progress}

After 30 years of the beginning of the first reverberation mapping analysis, our knowledge of the AGN physics has progressed significantly. And, although $\sim 100$ of sources have been monitored with this technique, their results have established the base for many other areas of AGN research. However, AGN with peculiar properties, like high accretion rate, have questioned their 

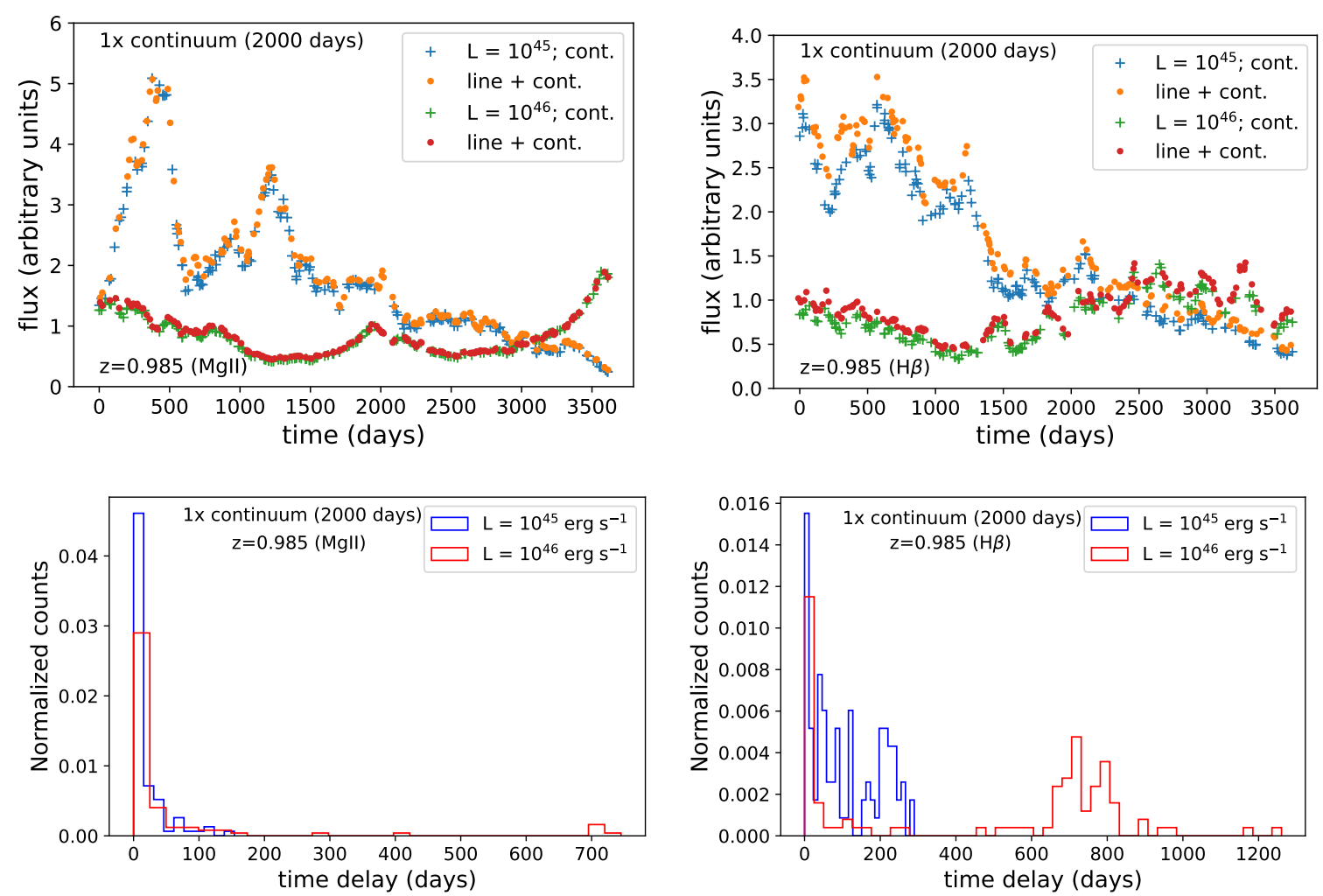

Figure 5: Preliminary lightcurves for MgII (UPPER-LEFT) and H $\beta$ (UPPER-RIGHT) at $z=0.985$, with arbitrary normalization. The corresponding time delay distributions are shown in the lower panels. The representation is same as Figure 4.

generalization of the $R_{\mathrm{H} \beta}-L_{5100}$ to the rest of AGN population. In this paper, we demonstrated that a correction can be applied to recover the expected $R_{\mathrm{H} \beta}-L_{5100}$ results. This correction is dependent on the chosen parameters, like the virial factor that is still under debate, but gives the first hints to understand and solve the problem. Upcoming LSST data will also test all these results, giving a statistical confirmation or generating new questions.

The code in development, although at a basic stage, is able to produce decent estimates of timedelay measurements. We are currently working to extend the analyses to include all six photometric channels simultaneously, and retrieve the time delay measurements to mimic actual observations. We have also tested the effect of having a denser coverage of photometric data points by scaling the continuum and the line contributions (Panda et al., 2019a) where we are now able to estimate the time-delays with an accuracy within 1-3\% error. The gain in accuracy is due to the fact that we incorporate a higher density of the continuum and the line contribution that is representative of the Deep-Drilling Fields (DDFs) in the LSST survey that will provide denser lightcurves compared to the rest of the All-Sky survey, as shown in Figure 10 in Panda et al. (2019a). We have also begun testing with different methodologies to generate lightcurves e.g. Damped-Random Walk and JAVELIN (Zu et al., 2011b, 2013, 2016). This also applies to the time-delay estimation where we are testing the consistency of the predicted values with other available methods e.g. Interpolated cross-correlation function (Gaskell and Peterson, 1987) and JAVELIN (Zu et al., 2011b, 2013, 
2016). Another property that should be taken into consideration is the potential contribution of the jet and its components to the optical luminosity, which can affect the positioning of the source within $R_{\mathrm{H} \beta}-L_{5100}$ relation in terms of the luminosity. In particular, correlations have been found between the radio spectral slope, radio loudness, the luminosity of [OIII] line and the strength of FeII line (Zajaček et al., 2019; Laor et al., 2019). More refined results will be presented in the near future.

\section{References}

Bentz, M. C., Denney, K. D., Grier, C. J., Barth, A. J., Peterson, B. M., Vestergaard, M., Bennert, V. N., Canalizo, G., De Rosa, G., Filippenko, A. V., Gates, E. L., Greene, J. E., Li, W., Malkan, M. A., Pogge, R. W., Stern, D., Treu, T., and Woo, J.-H.: 2013, ApJ, 767, 149

Brandt, W. N., Ni, Q., Yang, G., Anderson, S. F., Assef, R. J., Barth, A. J., Bauer, F. E., Bongiorno, A., Chen, C. T., De Cicco, D., Gezari, S., Grier, C. J., Hall, P. B., Hoenig, S. F., Lacy, M., Li, J., Luo, B., Paolillo, M., Peterson, B. M., Popović, L. C., Richards, G. T., Shemmer, O., Shen, Y., Sun, M., Timlin, J. D., Trump, J. R., Vito, F., and Yu, Z.: 2018, arXiv e-prints, p. arXiv:1811.06542

Brandt, W. N. and Vito, F.: 2017, Astronomische Nachrichten, 338(241), 241

Collin, S., Kawaguchi, T., Peterson, B. M., and Vestergaard, M.: 2006, A\&A, 456, 75

Czerny, B., Olejak, A., Rałowski, M., Kozłowski, S., Loli Martinez Aldama, M., Zajacek, M., Pych, W., Hryniewicz, K., Pietrzyński, G., Sobrino Figaredo, C., Haas, M., Średzińska, J., Krupa, M., Kurcz, A., Udalski, A., Gorski, M., Karas, V., Panda, S., Sniegowska, M., Naddaf, M.-H., Bilicki, M., and Sarna, M.: 2019, ApJ, 880(1), 46

Du, P., Hu, C., Lu, K.-X., Huang, Y.-K., Cheng, C., Qiu, J., Li, Y.-R., Zhang, Y.-W., Fan, X.-L., Bai, J.-M., Bian, W.-H., Yuan, Y.-F., Kaspi, S., Ho, L. C., Netzer, H., Wang, J.-M., and SEAMBH Collaboration: 2015, ApJ, 806, 22

Du, P. and Wang, J.-M.: 2019, ApJ 886(1), 42

Du, P., Zhang, Z.-X., Wang, K., Huang, Y.-K., Zhang, Y., Lu, K.-X., Hu, C., Li, Y.-R., Bai, J.-M., Bian, W.-H., Yuan, Y.-F., Ho, L. C., Wang, J.-M., and SEAMBH Collaboration: 2018, ApJ, 856, 6

Ferrarese, L. and Merritt, D.: 2000, ApJL, 539(1), L9

Gaskell, C. M. and Peterson, B. M.: 1987, ApJS, 65, 1

Grier, C. J., Shen, Y., Horne, K., Brandt, W. N., Trump, J. R., Hall, P. B., Kinemuchi, K., Starkey, D., Schneider, D. P., Ho, L. C., Homayouni, Y., I-Hsiu Li, J., McGreer, I. D., Peterson, B. M., Bizyaev, D., Chen, Y., Dawson, K. S., Eftekharzadeh, S., Guo, Y., Jia, S., Jiang, L., Kneib, J.-P., Li, F., Li, Z., Nie, J., Oravetz, A., Oravetz, D., Pan, K., Petitjean, P., Ponder, K. A., Rogerson, J., Vivek, M., Zhang, T., and Zou, H.: 2019, arXiv e-prints, p. arXiv:1904.03199 
Grier, C. J., Trump, J. R., Shen, Y., Horne, K., Kinemuchi, K., McGreer, I. D., Starkey, D. A., Brandt, W. N., Hall, P. B., Kochanek, C. S., Chen, Y., Denney, K. D., Greene, J. E., Ho, L. C., Homayouni, Y., I-Hsiu Li, J., Pei, L., Peterson, B. M., Petitjean, P., Schneider, D. P., Sun, M., AlSayyad, Y., Bizyaev, D., Brinkmann, J., Brownstein, J. R., Bundy, K., Dawson, K. S., Eftekharzadeh, S., Fernandez-Trincado, J. G., Gao, Y., Hutchinson, T. A., Jia, S., Jiang, L., Oravetz, D., Pan, K., Paris, I., Ponder, K. A., Peters, C., Rogerson, J., Simmons, A., Smith, R., and Wang, R.: 2017, ApJ, 851, 21

Gültekin, K., Richstone, D. O., Gebhardt, K., Lauer, T. R., Tremaine, S., Aller, M. C., Bender, R., Dressler, A., Faber, S. M., Filippenko, A. V., Green, R., Ho, L. C., Kormendy, J., Magorrian, J., Pinkney, J., and Siopis, C.: 2009, ApJ, 698(1), 198

Ivezić, Ž., Kahn, S. M., Tyson, J. A., Abel, B., Acosta, E., Allsman, R., Alonso, D., AlSayyad, Y., Anderson, S. F., Andrew, J., and et al.: 2019, ApJ, 873, 111

Kollmeier, J. A., Zasowski, G., Rix, H.-W., Johns, M., Anderson, S. F., Drory, N., Johnson, J. A., Pogge, R. W., Bird, J. C., Blanc, G. A., Brownstein, J. R., Crane, J. D., De Lee, N. M., Klaene, M. A., Kreckel, K., MacDonald, N., Merloni, A., Ness, M. K., O’Brien, T., Sanchez-Gallego, J. R., Sayres, C. C., Shen, Y., Thakar, A. R., Tkachenko, A., Aerts, C., Blanton, M. R., Eisenstein, D. J., Holtzman, J. A., Maoz, D., Nandra, K., Rockosi, C., Weinberg, D. H., Bovy, J., Casey, A. R., Chaname, J., Clerc, N., Conroy, C., Eracleous, M., Gänsicke, B. T., Hekker, S., Horne, K., Kauffmann, J., McQuinn, K. B. W., Pellegrini, E. W., Schinnerer, E., Schlafly, E. F., Schwope, A. D., Seibert, M., Teske, J. K., and van Saders, J. L.: 2017, arXiv e-prints, p. arXiv:1711.03234

Laor, A., Baldi, R. D., and Behar, E.: 2019, MNRAS 482(4), 5513

Luo, B., Brandt, W. N., Xue, Y. Q., Lehmer, B., Alexander, D. M., Bauer, F. E., Vito, F., Yang, G., Basu-Zych, A. R., Comastri, A., Gilli, R., Gu, Q. S., Hornschemeier, A. E., Koekemoer, A., Liu, T., Mainieri, V., Paolillo, M., Ranalli, P., Rosati, P., Schneider, D. P., Shemmer, O., Smail, I., Sun, M., Tozzi, P., Vignali, C., and Wang, J. X.: 2017, ApJS, 228(1), 2

Martínez-Aldama, M. L., Czerny, B., Kawka, D., Karas, V., Zajaček, M., and Życki, P. T.: 2019, Accepted for publication in ApJ, p. arXiv:1903.09687

Martínez-Aldama, M. L., del Olmo, A., Marziani, P., Sulentic, J. W., Negrete, C. A., Dultzin, D., D’Onofrio, M., and Perea, J.: 2018, A\&A, 618, A179

Marziani, P., Sulentic, J. W., Zwitter, T., Dultzin-Hacyan, D., and Calvani, M.: 2001, ApJ, 558, 553

Mejía-Restrepo, J. E., Lira, P., Netzer, H., Trakhtenbrot, B., and Capellupo, D. M.: 2018, Nature Astronomy, 2, 63

Negrete, C. A., Dultzin, D., Marziani, P., and Sulentic, J. W.: 2012, ApJ, 757(1), 62

Onken, C. A., Ferrarese, L., Merritt, D., Peterson, B. M., Pogge, R. W., Vestergaard, M., and Wandel, A.: 2004, ApJ, 615(2), 645 
Panda, S., Martínez-Aldama, M. L., and Zajaček, M.: 2019a, Frontiers in Astronomy and Space Sciences 6, 75

Panda, S., Marziani, P., and Czerny, B.: 2019b, ApJ 882(2), 79

Planck Collaboration: 2018, arXiv e-prints: p. arXiv: 1807.06209

Risaliti, G. and Lusso, E.: 2015, ApJ, 815, 33

Rodríguez-Pascual, P. M., Alloin, D., Clavel, J., Crenshaw, D. M., Horne, K., Kriss, G. A., Krolik, J. H., Malkan, M. A., Netzer, H., O’Brien, P. T., Peterson, B. M., Reichert, G. A., Wamsteker, W., Alexander, T., Barr, P., Blandford, R. D., Bregman, J. N., Carone, T. E., Clements, S., Courvoisier, T.-J., De Robertis, M. M., Dietrich, M., Dottori, H., Edelson, R. A., Filippenko, A. V., Gaskell, C. M., Huchra, J. P., Hutchings, J. B., Kollatschny, W., Koratkar, A. P., Korista, K. T., Laor, A., MacAlpine, G. M., Martin, P. G., Maoz, D., McCollum, B., Morris, S. L., Perola, G. C., Pogge, R. W., Ptak, R. L., Recondo-González, M. C., Rodríguez-Espinoza, J. M., Rokaki, E. L., Santos-Lleó, M., Sekiguchi, K., Shull, J. M., Snijders, M. A. J., Sparke, L. S., Stirpe, G. M., Stoner, R. E., Sun, W.-H., Wagner, S. J., Wanders, I., Wilkes, J., Winge, C., and Zheng, W.: 1997, ApJS, 110, 9

Sánchez-Sáez, P., Lira, P., Mejía-Restrepo, J., Ho, L. C., Arévalo, P., Kim, M., Cartier, R., and Coppi, P.: 2018, ApJ, 864, 87

Shen, Y. and Ho, L. C.: 2014, Nature 513, 210

Shen, Y., Richards, G. T., Strauss, M. A., Hall, P. B., Schneider, D. P., Snedden, S., Bizyaev, D., Brewington, H., Malanushenko, V., Malanushenko, E., Oravetz, D., Pan, K., and Simmons, A.: 2011, ApJS, 194, 45

Storchi-Bergmann, T., Schimoia, J. S., Peterson, B. M., Elvis, M., Denney, K. D., Eracleous, M., and Nemmen, R. S.: 2017, ApJ, 835(2), 236

Timmer, J. and Koenig, M.: 1995, A\&A, 300, 707

Wang, J.-M., Du, P., Hu, C., Netzer, H., Bai, J.-M., Lu, K.-X., Kaspi, S., Qiu, J., Li, Y.-R., Wang, F., and SEAMBH Collaboration: 2014a, ApJ, 793, 108

Wang, J.-M., Qiu, J., Du, P., and Ho, L. C.: 2014b, ApJ, 797, 65

Woo, J.-H., Yoon, Y., Park, S., Park, D., and Kim, S. C.: 2015, ApJ, 801, 38

Yu, L.-M., Bian, W.-H., Wang, C., Zhao, B.-X., and Ge, X.: 2019, arXiv e-prints, p. arXiv:1907.00315

Yu, L.-M., Zhao, B.-X., Bian, W.-H., Wang, C., and Ge, X.: 2020a, MNRAS 491(4), 5881

Yu, Z., Kochanek, C. S., Peterson, B. M., Zu, Y., Brandt, W. N., Cackett, E. M., Fausnaugh, M. M., and McHardy, I. M.: 2020b, MNRAS 491(4), 6045 
Zajaček, M., Busch, G., Valencia-S., M., Eckart, A., Britzen, S., Fuhrmann, L., Schneeloch, J., Fazeli, N., Harrington, K. C., and Zensus, J. A.: 2019, A\&A, 630, A83

Zu, Y., Kochanek, C. S., Kozłowski, S., and Peterson, B. M.: 2016, ApJ, 819, 122

Zu, Y., Kochanek, C. S., Kozłowski, S., and Udalski, A.: 2013, ApJ, 765, 106

Zu, Y., Kochanek, C. S., and Peterson, B. M.: 2011a, ApJ 735(2), 80

Zu, Y., Kochanek, C. S., and Peterson, B. M.: 2011b, ApJ, 735, 80

\section{DISCUSSION}

JAMES BEALL: What plan do you have for analyzing such volume of data?

MARY LOLI MARTÍNEZ-ALDAMA: We will focus on sources with spectroscopic measurements. LSST will be assisted by an array of campaigns (Brandt and Vito, 2017) during its proposed run. Moreover, there is a need for follow-up spectroscopic campaigns i.e. SDSS-V Black Hole Mapper (Kollmeier et al., 2017), which aim to derive BLR properties and reliable SMBH masses for distant AGNs with expected observed-frame reverberation lags of 10-1000 days. The SDSS-V Black Hole Mapper survey will also perform reverberation mapping campaigns in three out of the four LSST DDFs. These spectroscopic follow-up observations will allow us to choose the sources with better quality. With our code, we intend to prepare not only a mock catalog that will mimic the real observations that will be done by LSST, but also, use the real data to correct them that will then be user-ready for subsequent analyses. For more details about the data pipelines using LSST see Ivezić et al. (2019). 